\title{
Selenium and thyroid autoimmunity
}

\author{
Roberto Negro \\ Department of Endocrinology, \\ "V. Fazzi" Hospital, Lecce, Italy
}

Correspondence: Roberto Negro

Department of Endocrinology, "V. Fazzi"

Hospital, Piazza F. Muratore, 73100 ,

Lecce, Italy

Email robnegro@tiscali.it

\begin{abstract}
The trace element selenium (Se) occurs in the form of the amino acid selenocysteine in selenoproteins. Selenoproteins exerts multiple physiological effects in human health, many of which are related with regulation of reduction-oxidation processes. In fact, the selenoenzyme families of glutathione peroxidase (GPx) and thioredoxin reductase (TRx) display the ability to act as antioxidants, protecting cells from oxidative damage. Furthermore, another class of selenoproteins are the iodothyronine deiodinase enzymes (DIO), which catalyze the conversion of thyroxine (T4) in triiodothyronine (T3), then exerting a fine tuned control on thyroid hormones metabolism. Several studies have investigated the potential positive effects of Se supplementation in thyroid diseases, characterized by increased levels of hydrogen peroxide and free radicals, like autoimmune chronic thyroiditis. These studies have supplied evidences indicating that Se supplementation, maximizing the antioxidant enzymes activity, may reduce the thyroid inflammatory status. Then, it may be postulated that Se could play a therapeutical role in thyroid autoimmune diseases. Despite the fact that recent studies seem to be concordant about Se beneficial effects in decreasing thyroid peroxidase antibodies (TPOAb) titers and ameliorating the ultrasound echogenicity pattern, several doubts have to be still clarified, before advising Se supplementation in chronic autoimmune thyroiditis.
\end{abstract}

Keywords: selenium, thyroid, autoimmunity

\section{Introduction}

The trace element selenium (Se) was discovered in 1817 by the Swedish chemist Berzelius. Two hundred years later, Se is well-known as an essential mineral of pivotal importance for the human health. It is established that Se displays antioxidant activity, antiinflammatory chemopreventive, and antiviral characteristics (Rayman 2000). Its effect on human health is due to its presence within some proteins (Kryukov et al 2003). Unlike other metal elements that interact with proteins in form of cofactors, Se becomes cotranslationally incorporated into polypeptide chain as part of the amino acid selenocysteine (Sec), also known as the 21 th aminoacid (Gromer et al 2005). The group of proteins that contain Sec as an integral part of their polypeptide chain are defined as selenoproteins. Between the domains, 25 selenoprotein families have been identified in humans (Castellano et al 2005). The human selenoproteome is composed of 17 selenoprotein families, some with multiple genes having similar functions. These comprise glutathione peroxidase (GPx) (five genes), thioredoxin reductase (TrxR) (three genes), and iodothyronine deiodinases (DIO) (three genes), which have been functionally characterized as having oxidoreductase functions (Kryukov et al 2003). The entry point of Se in animals is via plants, which absorb the element in its organic form from the soil, where its content varies between different areas. In plants, Se becomes converted to organic forms such as methylated low-molecular-weight Se compounds and the amino acid selenomethionine (SeMet) and Sec (Whanger 2002). Importantly, Se compounds have the capacity to redox cycle and are metabolized to more reduced states, which are thought to account for Se compounds being effective antioxidants. In fact, Sec is part of the catalytic group within selenoenzymes and is directly involved 
in redox reactions (Zhong and Holmgren 2000). The effects of Se on the organism are concentration-dependent, ranging from an antioxidant activity in the nanomolar-micromolar range to potentially prooxidant at concentrations above what is required for maximal selenoprotein synthesis. At even higher concentrations, Se compounds may accumulate and redox cycle with intracellular thiols, inducing oxidative stress and cellular damage (Vinceti et al 2001). Se is essential for life, and there is no doubt that adequate amounts of this element are required for optimal human health. Many of its physiologic roles may be ascribable to its presence within selenoproteins. For example, one of the most important cellular processes, DNA synthesis, depends on the presence of Se within the catalytic site of TrxR (Arner and Holmgren 2000). Moderate Se deficiency has been associated to many conditions, such as increased risk of cancer and infections, male infertility, increased thyreocytes damage, and serious neurological diseases, including Alzheimer's and Parkinson's (Rayman 2000). However, for some of these conditions, the evidence is rather scant, conflicting data have been published, and need further confirmations. On the contrary, a pathological condition that is certainly associated with Se deficiency is Keshan disease, a potentially fatal form of cardiomyopathy; this disease is prevalent in children and is endemic in some areas of China characterized by extremely low levels of Se in the soil (Levander and Beck 1997). It is supposed that infection by Coxsackie B virus may trigger the onset of this disease. Notably, the condition is prevented or completely reversed by Se supplementation (Xia et al 2005). Another disease due to a combined deficiency of Se and iodine is myxedematous cretinism which is characterized by mental and growth retardation (Vanderpas et al 1990). It is ascertained that Se deficiency causes a reduction in GPx and DIO enzymes activity, accumulation of hydrogen peroxide $\left(\mathrm{H}_{2} \mathrm{O}_{2}\right)$ causing damage to the thyroid gland, and impaired thyroid hormone metabolism. Se supplementation in this condition must be administered after iodide levels have been restored, as Se increases the activity of DIO, leading to a further loss of iodide from the damaged thyroid (Zimmermann and Kohrle 2002).

In the United States, where dietary intakes are higher than in many other countries, the current recommendation is $55 \mu \mathrm{g} /$ day. In the United Kingdom, however, Se intake is considerably lower, and the recommended dose is $75 \mu \mathrm{g} /$ day for men and $60 \mu \mathrm{g} /$ day for women (Surai 2006). These recommendations were based on the plasma GPx optimal enzyme activity, however, a recent study indicates that higher Se intake is required to obtain full expression of selenoprotein P (SelP) (Xia et al 2005). Then, it is suggested that SelP may be a better indicator of Se nutritional status than GPx, and that the recommended dietary intake may need to be revised.

Even if a large body of studies has made available a vast information about Se, the precise molecular mechanisms behind its effects in physiologic and pathologic conditions remain unknown.

\section{The best known and most important human selenoproteins}

The most common form of Se in human proteins is Sec, which becomes cotranslationally incorporated within the growing polypeptide chain. However, selenium-containing proteins exists also in other forms, such as proteins that nonspecifically incorporate Se during translation, and selenium-binding proteins that bind Se as a cofactor. Only a few of the 25 identified mammalian selenoproteins have so far been functionally characterized. Most of these selenoproteins exhibit enzymatic redox function via Sec, which confers their catalytic and antioxidant activities (Castellano et al 2005).

\section{Thioredoxin reductase}

Thioredoxin reductase (TrxR) and NADPH constitute the thioredoxin system, a major cellular redox system present in all living organisms (Arner and Holmgren 2000). Three mammalian TrxR selenoenzymes have been identified: the cytosolic enzyme (TrxR1), the mitochondrial enzyme (TrxR2), and a testis-specific enzyme thioredoxingluthatione reductase (TrxR3) (Sun et al 1999, 2001). The Trx system plays a pivotal role in embryo development. Knockout mouse model for Trx gene (Txn) are not vital and homozygous mutants for TrxR1 die shortly after implantation as a result of failure to proliferate, suggesting that TrxR is essential for early differentiation and morphogenesis (Matsui et al 1996; Jakupoglu et al 2005). The similar Txn knockout and TrxR1 homozygous mutants phenotypes are mostly likely explained by impaired DNA synthesis due to accumulation of oxidized, nonfunctional ribonucleotide reductase. These results confirm that the TrxR system is absolutely required for development, and probably cell proliferation in vivo, and also emphasize the importance of the Sec residue for catalytic activity. As observed for the TrxR1, complete removal of mitochondrial TrxR2 causes embryonic death and homozygous mutant embryos show decreased hematopoiesis, increased apoptosis in the liver, and cardiac defects (Nonn et al 2003; Conrad et al 2004). 


\section{Glutathione peroxidases (GPx)}

Glutathione peroxidases (GPx) was the first mammalian protein shown to incorporate $\mathrm{Se}$ in the form of $\mathrm{Sec}$ into catalytic site and was supposed to be associated with the antioxidant activity of Se. GPxs are recognized for catalyzing the reduction of hydrogen peroxide and organic hydroperoxides, thus protecting cells from oxidative damage. The importance of glutathione peroxidases and their potentially beneficial role in critically ill patients concerns the mechanism of detoxification of peroxides to their respective alcohols at the expense of glutathione. It seems that all of the glutathione peroxidase isoforms share the same catalytic mechanism, with a highly conserved sequence of selenocysteine, tryptophan and glutamine (Aumann et al 1997; Brigelius-Flohe 1999). The role of plasma GPx as an antioxidant enzyme is not fully understood. Although its preferred substrate, glutathione, usually has a low plasma concentration, increased cellular oxidative stress can export oxidized glutathione to the plasma compartment, where a combination of glutathione reductase and GPx can restore redox balance and either return reduced glutathione to the cell, or use it as a substrate for plasma GPx. Thus, Se can act as antioxidant when incorporated as selenoenzymes in the extracellualr space, the cell cytosol, in association with cell membranes, having the potential role to influence the immune processes (Arthur 2000). Localization and main function of the seven GPx isoenzymes are resumed in Table 1.

\section{lodothyronine deiodinases}

Three differentially distributed Sec-containing oxidoreductases (DIO1, DIO2, DIO3) constitute the family of iodothyronine deiodinases (DIO). They catalyze the activation (DIO1 and DIO2) and inactivation (DIO3) of the thyroid hormones thyroxine (T4), 3,5,3'- triodothyronine (T3), and reverse-3,5,3'-triiodothyronine (rT3) by removing distinct iodine moieties, as indicated in Figure 1 (Bianco and Kim 2006). Among their actions, thyroid hormones contribute to regulate the function of heart, metabolism, growth, are pivotal for the development of the fetal brain, and display multiple interactions with other hormonal systems. DIOs exhibit different localizations and function in human tissues (St Germain et al 2005). DIO1 is expressed mostly in the liver, kidney, thyroid, and pituitary; DIO2 in the thyroid, central nervous system, pituitary, and skeletal muscle; DIO3 is most prominently expressed in the pregnant uterus, placenta, embryonic liver, embryonic and neonatal brain, and neonatal skin. The three DIOs exert different actions as DIO1 plays a role in $\mathrm{T} 3$ production in the thyroid

Table I Localization and function of glutathione peroxidase (Stawicki et al 2007)

\begin{tabular}{|c|c|c|}
\hline $\begin{array}{l}\text { Glutathione peroxidase } \\
\text { isoenzyme }\end{array}$ & Localization & Function \\
\hline GPx I & Liver, erytrocytes & $\begin{array}{l}\text { Important in severe oxidative stress. Role in viral } \\
\text { infectious processes }\end{array}$ \\
\hline GPx 2 & Liver, gastrointestinal tract & $\begin{array}{l}\text { First line of defence against ingested organic } \\
\text { hydroperoxides. Involvement in apoptosis, cellular } \\
\text { proliferation }\end{array}$ \\
\hline GPx 3 & $\begin{array}{l}\text { Plasma, intestine, breast milk, adrenal gland, } \\
\text { pulmonary lavage fluid, renal proximal } \\
\text { tubules }\end{array}$ & $\begin{array}{l}\text { Thought to have largely regulatory functions } \\
\text { associated with oxidative stress and malignancy. } \\
\text { Expression induced by hypoxia }\end{array}$ \\
\hline GPx 4 & $\begin{array}{l}\text { Distributed in cytoplasm, nucleus and mito- } \\
\text { chondria. Expressed in testis, lung, heart, } \\
\text { cerebellum. Essential structural component } \\
\text { of the sperm's midpiece }\end{array}$ & $\begin{array}{l}\text { Antioxidant involved in protection of biomem- } \\
\text { branes. Reduces phospholipids hydroperoxides in } \\
\text { cellular membranes. Involved in redox signalling } \\
\text { and regulatory processes (apoptosis, inhibition of } \\
\text { lipoxygenases). Required for sperm fertilization. } \\
\text { Lack of GPx } 4 \text { is lethal at an early embyogenic } \\
\text { stage }\end{array}$ \\
\hline GPx 5 & Epididymis & $\begin{array}{l}\text { Potentially serves a "backup" function for seleno- } \\
\text { cysteine containing GPx isoforms in sperm. Can } \\
\text { be secreted or membrane-bound }\end{array}$ \\
\hline GPx 6 & $\begin{array}{l}\text { Bowman's gland, olfactory epithelium, } \\
\text { embryonic tissues }\end{array}$ & Olfaction. Putative odorant metabolizing enzyme \\
\hline GPx 7 & Breast tissues & Breast cancer defense against oxidative stress \\
\hline
\end{tabular}

Abbreviation: GPx, glutathione peroxidases. 


\section{Thyroxine (T4)}
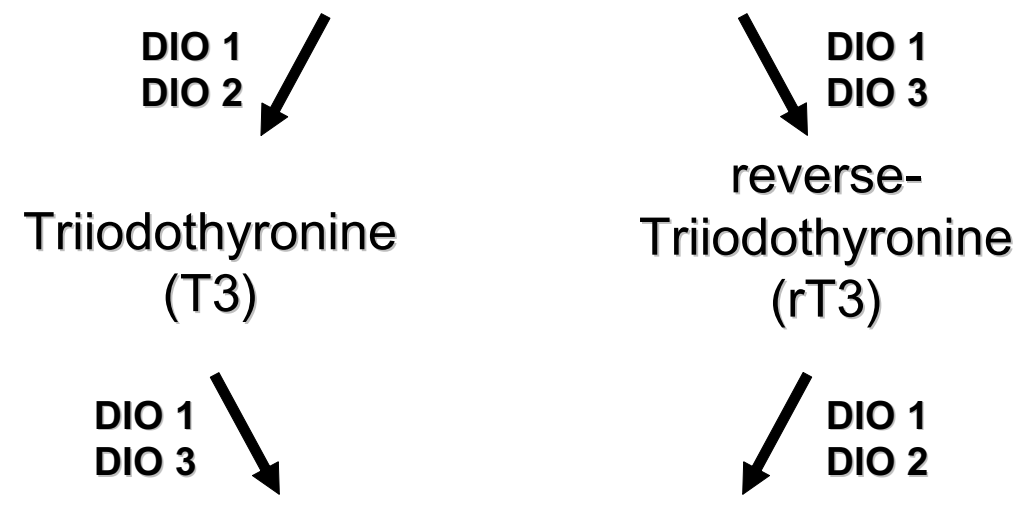

Diiodothyronine (T2)

Figure I Metabolism of thyroid hormones by types I, 2, and 3 deiodinases.

gland and controls the circulating T3 levels, whereas DIO2 and DIO3 are proposed to function in the local deiodination processes. Recent evidence indicates that DIO2 represents a major source of circulating T3 in euthyroid humans, whereas DIO1 contributes more substantially at high, thyrotoxic T4 levels, as seen in hyperthyroid patients. The presence of $\mathrm{Sec}$ appears to be absolutely required for DIO catalytic activity and thus it is not surprising that Se levels have a direct regulatory effect on the expression of DIOs (Bates et al 2000; Kohrle et al 2005). Among various selenoproteins, DIOs rank high in the hierarchy of Se supply during Se deficiency, DIO1 expression being maintained or slightly increased in the thyroid gland. In the brain and placenta, DIO1 and DIO3 expression is maintained during Se restriction, whereas DIO1 expression decreases in other tissues. The mechanisms of DIOs regulation by Se availability is not fully understood, nevertheless, it appears to be tissue- or organ-specific. Additional regulators of DIOs expression are T3 and thyrotropin (TSH) (which act in a feedback loop), and cyclic adenosine monophosphate (cAMP) (Koenig 2005). Experimental models with knockout transgenic mouse, provided evidence of the crucial roles exerted by DIOs. In particular, knockout mice for DIO1 display abnormal concentrations of thyroid hormones and their metabolities, suggesting a role in preserving iodine store within the organism; DIO2 deficient mice have alterations in auditory function, thermogenesis, and brain development; DIO3 knockout models exhibit reduced viability, growth retardation, impaired fertility, reduced T3 and increased T4 levels (St Germain et al 2005). There are not well documented diseases derived from primary DIOs deficiency in humans. However, in 2005, mutations of Sec binding protein 2 have been identified. The authors reported an inherited selenocysteine defect, caused by a homozygous misense mutation of SBP2, which is an indispensable protein for selenoprotein synthesis, resulting in abnormalities in the deiodinases and defective thyroid metabolism (Dumitrescu et al 2005). Several diseases with disturbed thyroid hormone metabolism and expression of DIOs are known. In Graves' hyperthyroidism the increased levels of $\mathrm{T} 3$ are known to induce DIO1 expression at the transcriptional level, which further contributes to the progression of the disease; on the other hand the use of propylthiouracil (PTU) inhibits DIO1 by binding to Sec residue, which represents a benefit in the treatment of hyperthyroidism (Bianco et al 2002; Leonard and Rosenberg 2003). In hypothyroidism, decreased DIO1 levels with a concomitant increased DIO2 activity may be observed, potentially to serve as a rescue mechanism to provide local levels of T3 (Bianco et al 2002). Combined Se and iodine deficiency leads to the condition of myxedematous cretinism. Reduced DIOs and GPx activity are both supposed to play a central role for the development of this disease. The decreased GPx activity increase oxidative damage in the thyroid, while the reduced DIO concentration profoundly alters thyroid hormone metabolism (Kohrle et al 2005). The involvement of Se and selenoproteins in autoimmune thyroid diseases are discussed in a separate paragraph.

\section{Other selenoproteins}

Several others selenoproteins have been identified, and their localizations and functions have been quite clearly 
defined. They are involved in multiple processes that concern transport and delivery of Se, apoptosis, proliferation and cancer, muscle cells function, inflammation, and oxidation (see Table 2). Furthermore, there are some others selenoproteins that have not been characterized (SelH, SelO, SelT, and SelV), and whose functions are thought to be related to redox processes (Castellano et al 2005).

\section{Selenium and thyroid metabolism}

The importance of Se for the thyroid function has been increasingly recognized and multiple laboratory experiments, clinical trials, and epidemiologic data have progressively revealed the relationships between $\mathrm{Se}$, iodine, and thyroid hormones metabolism. Of interest, for example, is the condition of iodine deficiency. This situation, which is associated with high TSH values, produce increased levels of $\mathrm{H}_{2} \mathrm{O}_{2}$ (Smyth 2003). The cytotoxic action on thyroid cells depends on both the formation of free radicals and the defence ability of antioxidative enzymes, the latter being impaired in a state of Se deficiency and altered selenoproteins activity (Ekholm and Bjorkman 1997). In order to accept electrons derived from oxidative reactions, the thyroid cells physiologically generate a large amount of $\mathrm{H}_{2} \mathrm{O}_{2}$ at the cell surface. The thyroid is thus a source of $\mathrm{H}_{2} \mathrm{O}_{2}$, a necessary substrate for thyroperoxidase (TPO) activity. The generation of $\mathrm{H}_{2} \mathrm{O}_{2}$ is the rate-limiting step in the thyroid hormone synthesis and is regulated by TSH action on an interacting second-messenger system (Kimura et al 1995). The production of $\mathrm{H}_{2} \mathrm{O}_{2}$ leads to the iodination of tyrosine residues and the coupling of iodinated tyrosines to thyroglobulin ( $\mathrm{Tg}$ ). It is known that the $\mathrm{Tg}$ iodination in vivo takes place on the external surface of the apical plasma membrane, where TPO is integrated and $\mathrm{H}_{2} \mathrm{O}_{2}$ is generated (Bjorkman and Erkhold 1995). $\mathrm{H}_{2} \mathrm{O}_{2}$ may easily cross the apical membrane to the luminal site where it reacts with TPO for the iodination of Tg. Then, when $\mathrm{H}_{2} \mathrm{O}_{2}$ is available, iodination may be catalyzed by TPO and controlled by GPx, which degrades $\mathrm{H}_{2} \mathrm{O}_{2}$. Excess $\mathrm{H}_{2} \mathrm{O}_{2}$ may diffuse into the cell, where it will promptly be attacked by GPx, TRx, and catalase, which is present in the peroxisomes (Corvilain et al 2000). Increased GPx activity reduces $\mathrm{H}_{2} \mathrm{O}_{2}$ availability, whereas in conditions of Se deficiency decreased GPx activity results in higher generation of $\mathrm{H}_{2} \mathrm{O}_{2}$ and increased TPO activity. Therefore, the expected activity of the GPx system plays a pivotal function in the iodination process, while, the intrathyroidal concentration of Se is important for GPx activity. The GPx and catalase antioxidant systems are protected by the antioxidant protein thiol-specific antioxidant (TSA), which modulates $\mathrm{H}_{2} \mathrm{O}_{2}$-mediated responses. TSA is TSH-dependent regulated, although the effect of TSH on TSA gene expression at the transcriptional level remains unclear (Kim et al 2001).

\section{Selenium and thyroid autoimmunity}

Some interventional studies have tested the hypothesis that Se administration may have a beneficial effect on autoimmune thyroiditis (AIT). It has recently been reported that in patients with AIT from an area in South Germany with borderline Se intake, $200 \mu \mathrm{g}$ 3-month Se supplementation, in the form of selenite, significantly reduced anti-TPOAb

Table 2 Localizations and functions of selenoproteins other than GPx, DIO, and TrxR

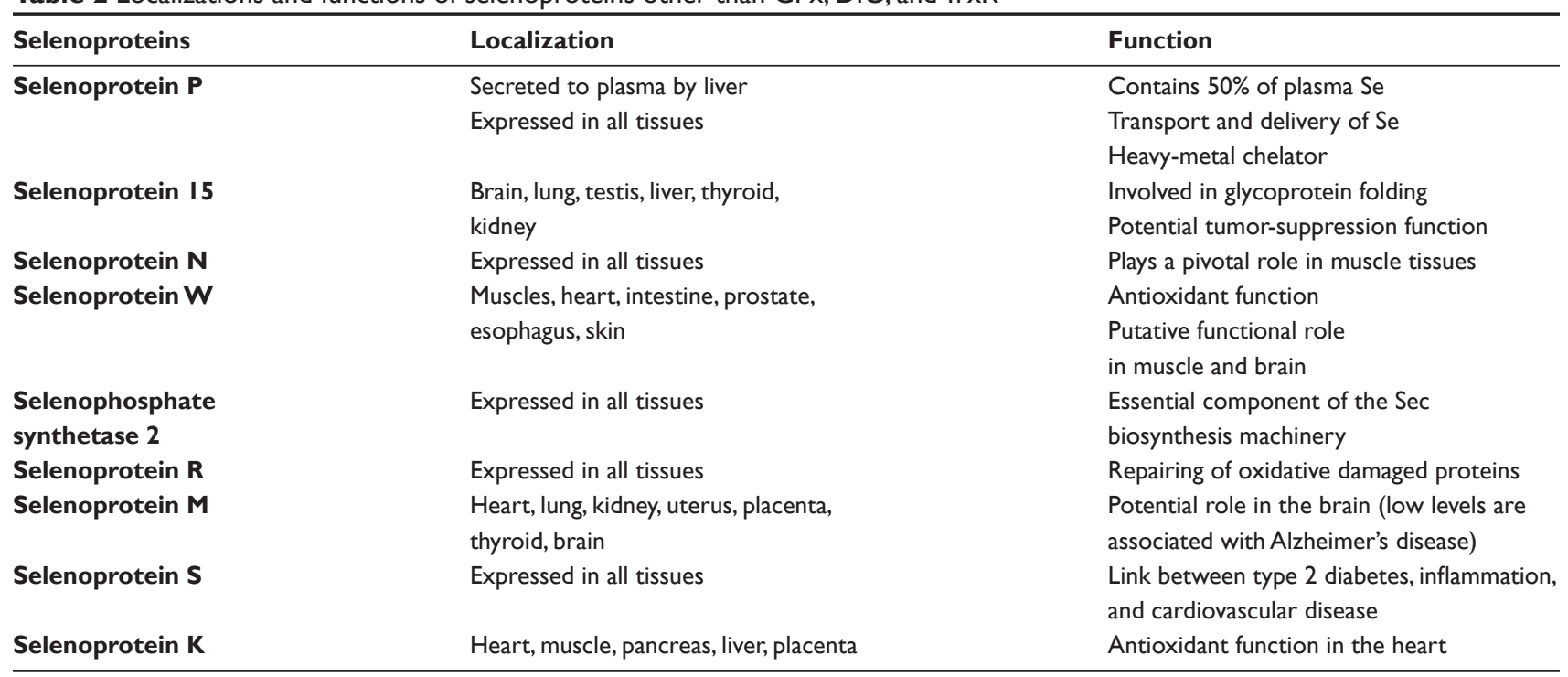

Abbreviation: Se, selenium. 
concentration and improved ultrasound echomorphology (Gärtner et al 2002). In a 6-month follow-up crossover study, the anti-TPO concentrations continued to decrease significantly in the group that received Se, whereas the anti-TPOAb titers significantly increased in the group that stopped taking Se (Gärtner and Gasnier 2003). These findings are supported by another study conducted in patients with AIT in a non-Se-deficient area in Greece, in which anti-TPOAb concentrations also significantly decreased with a combined treatment over 6 months of $200 \mu \mathrm{g}$ of selenomethionine and LT4 (Duntas et al 2003). Anti-TPOAb reduction was prominent in the first 3 months of treatment, which may be the result of elevated intrathyroidal Se levels achieved during the study with consequent enhancement of the scavenging activity of both the GPx and TRx systems. In this respect, supplemented Se protected against goiter and thyroid tissue damage in a study from France that included 792 men and 1108 women from the SU.VIMAX study (Derumeaux et al 2003). The authors also found a relationship to thyroid echostructure and concluded that Se may protect against autoimmune thyroid disease. Another recent study from Greece, evaluated the effects of Se supplementation (200 $\mu \mathrm{g} /$ day) in patients suffering from AIT. After 6 months of treatment a $9.9 \%$ reduction of TPOAb titers was observed; afterwards, while in one group the Se supplementation was continued, and a further decrement of TPOAb was observed, in the other group which stopped supplementation, a $4.8 \%$ increase took place (Mazopakis et al 2007). It has also been suggested that Se administration may have a protective role in pregnant women with AIT who are at a higher risk of miscarriage, as women with pregnancy loss had significantly lower Se hair content than controls (Al Kunani et al 2001; Prummel and Wiersinga 2004). A particularly interesting patients in the field of AIT, is represented by pregnant women. These patients are characterized by an increased risk of miscarriage, preterm delivery, and development of thyroid dysfunction after delivery (Negro et al 2006). One study, involving 2143 euthyroid pregnant women (7.9\% of them with TPOAb), evaluated the effects of $200 \mu \mathrm{g}$ of selenomethionine supplementation during and after pregnancy. Results showed that, in women with autoimmune chronic thyroiditis, post-partum thyroid dysfunction and permanent hypothyroidism were lower in the treated group in respect to the untreated group (28.6 vs. 48.6\%, $P<0.01$; and 11.7 vs. $20.3 \%, P<0.01$, respectively) (Negro et al 2007) (Figure 2). The same study demonstrated that the Se supplemented women displayed lower TPOAb titers during the postpartum period and better thyroid ultrasound patterns when compared with the untreated ones. Finally, Moncayo and Moncayo (2005) reported in a paper published in 2005 few cases of patients with autoimmune hypothyroidism who benefited from a daily Se supplementation. The cited

\section{Selenium Reduces PPTD and Hypothyroidism}

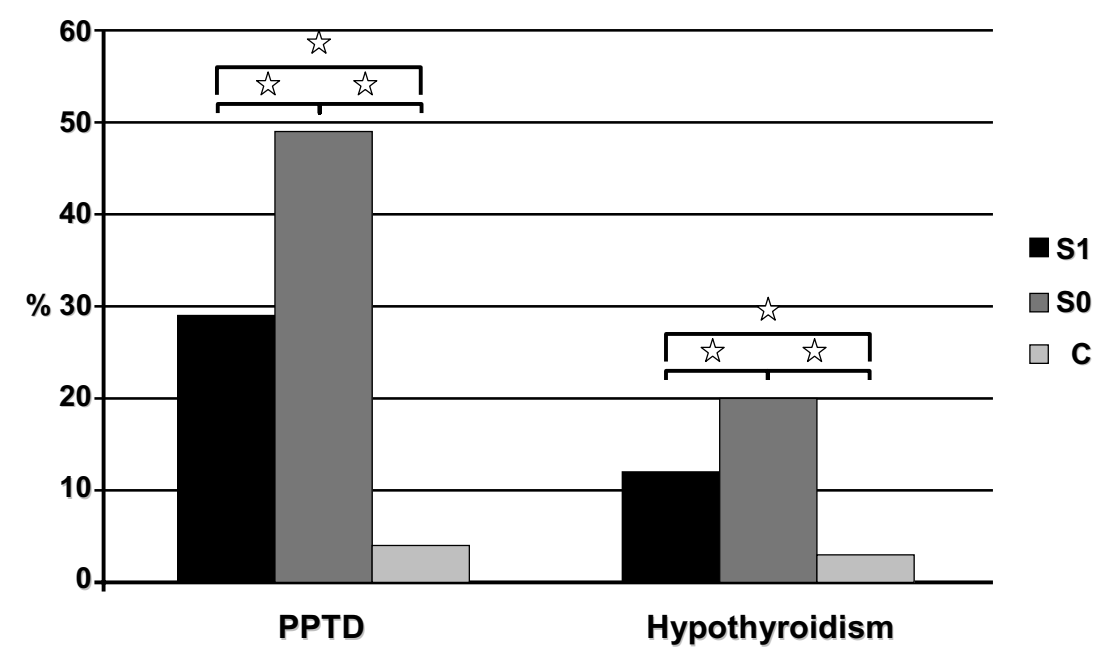

Figure 2 Percentage of patients who had PPTD (left) and hypothyroidism (right) develop in TPOAb(+) women who received Se (group SI) or placebo (group S0), and in TPOAb(-) women (group C)., $P<0.0$ l copyright (C) 2007. The Endocrine Society. Reproduced with permission from Negro R, Greco G, Mangieri T, et al. 2007. The influence of selenium supplementation on postpartum thyroid status in pregnant women with thyroid peroxidase autoantibodies.J Clin Endocrinol Metab, 92: $1263-8$. 
patients, having serum Se concentrations below the lower limit, had an extraordinary recovery of thyroid function after Se treatment, with a condition of restored euthyroidism and an ameliorated ultrasound echogenicity pattern (Moncayo and Moncayo 2005).

Despite their different phenotypes, Hashimoto's thyroiditis and Graves' disease (GD), share the production of organ-specific antibodies, and a common genetic background (Dayan and Daniels 1996; Strieder et al 2003; Caturegli et al 2007). GD is characterized by a condition of increased oxidative stress, not only in the acute phase of the disease, but also in the state of euthyroidism, induced by anti-thyroid medications (Ademog `lu et al 2006). In fact, several studies outlined the association of GD with impaired antioxidant activity. Urinary malondialdehyde levels, a marker of oxidative stress, is higher in hyperthyroid patients compared to euthyroid controls (Guerra et al 2005). In patients suffering from GD, the use of antithyroid drugs, either methimazole or propylthouracil, have been proven to be effective in lowering oxidant generation and improving the imbalance of the antioxidant/oxidant status (Seven et al 2001; Abalovich et al 2003). A recent study by Wertenbruch and colleagues (2007) compared serum Se levels in patients with remission and relapse of GD. The authors found that highest serum Se levels ( $>120$ microg/ 1) were seen in the remission group, indicating a positive effect of Se levels on the outcome of GD. In addition, the authors showed that TSH-receptor antibodies levels and serum Se values were positively correlated in the relapse group, whereas a negative correlation of both parameters were seen in the remission group, supporting the idea of a positive effect of Se on thyroidal autoimmune process (Wertenbruch et al 2007). One study from Croatia, evaluated the effects of supplementation with a fixed combination of antioxidants (vitamins $\mathrm{C}$ and $\mathrm{E}$, beta-carotene and selenium) on superoxide dismutase activity, copper and zinc concentrations, and total antioxidant status in erythrocytes derived from a group of patients with GD treated with methimazole, with respect to the rate of achieving euthyroidism (Bacic-Vrca et al 2005). Results showed that patients receiving antioxidant supplementation along with methimazole therapy achieved euthyroidism at a faster rate than those treated with methimazole alone. The activity of superoxide dismutase similarly decreased in both groups, while the total antioxidant status was mostly improved in the supplemented group. Taking together, the abovementioned studies' results, may lead to think that Se supplementation may exert a beneficial effect on the course of GD.

\section{Conclusion}

Se is a mineral of pivotal importance for human health. It is an integral part of selenoproteins, many of which are involved in redox processes and are effective as antioxidants. The most frequent disease involving the thyroid gland is the autoimmune chronic thyroiditis, an inflammatory process which progressively destroy the gland. Several studies have examined the potential benefits of Se in this disease to assess if its supplementation may be effective in increasing the antioxidant defences. These studies have by consent demonstrated that Se supplementation, probably maximizing the Se-dependent enzymes activity, is able to reduce the inflammatory state, exerting a decline of TPOAb titers and an amelioration of the ultrasound pattern. Furthermore, there are some preliminary data which indicate a possible role of Se supplementation in ameliorating the course of GD, even if specifically and well-designed randomized controlled trials are necessary to address this issue.

However, several points have to be still clarified. Firstly, we have not fully understood functions and actions of the already identified selenoproteins; then, the first question is whether the effect of Se on thyroid autoimmunity depends on the maximization of GPx activity alone or also of other selenoproteins. Secondly, it has still to be defined the exact amount of Se to be given to maximize the enzymatic antioxidant activity. Thirdly, a cost/benefit evaluation is mandatory; in fact, the final result of autoimmune chronic thyroiditis is represented by hypothyroidism; Se supplementation should delay the time of Levothyroxine initiation, but obliges in any case the patient to take a tablet a day, and even when hypothyroidism develops, substitutive treatment with Levothyroxine has no side effects and it is a very cheap drug. One viable application of Se, may be its supplementation during pregnancy (possibly in conjunction with iodine supplementation). In fact, by now, screening for thyroid function during pregnancy is not warranted (Abalovich et al 2007). Considering that up to $\sim 10 \%$ of pregnant women are positive for TPOAb, and that about $8 \%$ of all pregnant women develop postpartum thyroiditis, it is plausible a positive effect exerted by Se in this particular kind of patients (Poppe and Glinoer 2003; Stagnaro-Green 2004). Finally, it has to be reminded that Se have had a putative role in preventing atherosclerosis and carcinogenesis, and a possible action in improving glucose metabolism. All these hypothesis have been proven to be wrong, and in addition, a recently published study even suspected Se to be responsible for increased risk of developing diabetes (Hunter et al 1990; Garland et al 1995; Bleys et al 2006; Stranges et al 2007). 


\section{Disclosure}

Dr. Negro reports no conflicts of interest.

\section{References}

Abalovich M, Llesuy S, Gutierrez S, et al. 2003. Peripheral parameters of oxidative stress in Graves' disease: the effects of methimazole and 131 iodine treatments. Clin Endocrinol, 59:321-7.

Abalovich M, Amino M, Barbour LA et al. 2007. Management of thyroid dysfunction during pregnancy and postpartum: an endocrine society clinical practice guideline. J Clin Endocrinol Metab, 92:S1-S47.

Ademoğlu E, Ozbey N, Erbil Y, et al. 2006. Determination of oxidative stress in thyroid tissue and plasma of patients with Graves' disease. Eur J Intern Med, 17:545-50.

Al Kunani AS, Knight R, Haswell SJ, et al. 2001. The selenium status of women with a history of recurrent miscarriage. Br J Obstet Gynaecol, 108:1094-7.

Arner ES, Holmgren A. 2000. Physiological functions of thioredoxin and thioredoxin reductase. Eur J Biochem, 267:6102-9.

Arthur JR. 2000. The glutathione peroxidases. Cell Mol Life Sci, 55:1825-35.

Aumann KD, Bedorf N, Brigeluis-Flohe R, et al. 1997. Glutathione peroxidase revisited--simulation of the catalytic cycle by computer-assisted molecular modelling. Biomed Environ Sci, 10:136-55.

Bacic-Vrca V, Skreb F, Cepelak I, et al. 2005. The effect of antioxidant supplementation on superoxide dismutase activity, $\mathrm{Cu}$ and $\mathrm{Zn}$ levels, and total antioxidant status in erythrocytes of patients with Graves' disease. Clin Chem Lab Med, 43:383-8.

Bates JM, Spate VL, Morris JS, et al. 2000. Effects of selenium deficiency on tissue selenium content, deiodinase activity, and thyroid hormone economy in the rat during development. Endocrinology, 141:2490-500.

Bianco AC, Kim BW. 2006. Deiodinases: implications of the local control of the thyroid hormone action. $J$ Clin Invest, 116:2571-9.

Bianco AC, Salvatore D, Gereben B, et al. 2002. Biochemistry, cellular and molecular biology, and physiological roles of the iodothyronine selenodeiodinases. Endocr Rev, 23:38-89.

Bjorkman U, Ekholm R. 1995. Hydrogen peroxide degradation and glutathione peroxidase activity in cultures of thyroidal cells. Mol Cell Endocrinol, 111:99-107.

Bleys J, Miller ER3rd, Pastor-Barriuso R, et al. 2006. Vitamin-mineral supplementation and the progression of atherosclerosis: a meta-analysis of randomized controlled trials. Am J Clin Nutr, 84:880-7.

Brigelius-Flohe R. 1999. Tissue-specific functions of individual glutathione peroxidases. Free Radic Biol Med, 27:951-65.

Castellano S, Lobanov AV, Capple C, et al. 2005. Diversity and functional plasticity of eukaryotic selenoproteins: identification and characterization of the Sel family. Proc Natl Acad Sci USA, 102:16188-93.

Caturegli P, Kimura H, Rocchi R, et al. 2007. Autoimmune thyroid diseases. Curr Opin Rheumatol, 19:44-8.

Conrad M, Jakupoglu C, Moreno SG, et al. 2004. Essential role for mitochondrial thioredoxin reductase in hematopoiesis, heart development, and heart function. Mol Cell Biol, 24:9414-23.

Corvilain B, Collyn L, van Sande J, et al. 2000. Stimulation by iodide of $\mathrm{H} 2 \mathrm{O} 2$ generation in thyroid slices from several species. Am J Physiol Endocrinol Metab, 278:692-9.

Dayan CM, Daniels GH. 1996. Chronic autoimmune thyroiditis. $N$ Engl J Med, 335:99-107.

Derumeaux H, Valeix P, Castetbon K, et al. 2003. Association of selenium with thyroid volume and echostructure in 3-to 60-year-old French adults. Eur J Endocrinol, 148:309-15.

Dumitrescu AM, Liau XH, Abdullah MS, et al. 2005. Mutations in SECISBP2 result in abnormal thyroid hormone metabolism. Nat Genet, 37:1247-52.

Duntas LH, Mantzou E, Koutras DA. 2003. Effects of a six month treatment with selenomethionine in patients with autoimmune thyroiditis. Eur $J$ Endocrinol, 148:389-93.
Ekholm R, Bjorkman U. 1997. Glutathione peroxidase degrades intracellular hydrogen peroxide and thereby inhibits intracellular protein iodination in thyroid epithelium. Endocrinology, 138:2871-8.

Garland M, Morris JS, Stampfer MJ, et al. 1995. Prospective study of toenail selenium levels and cancer among women. J Natl Cancer Inst, $87: 497-505$

Gärtner R, Gasnier BC, Dietrich JW, et al. 2002. Selenium supplementation in patients with autoimmune thyroiditis decreases thyroid peroxidase antibodies concentrations. J Clin Endocrinol Metab, 87:1687-91.

Gärtner R, Gasnier BC. 2003. Selenium in the treatment of autoimmune thyroiditis. Biofactors, 19:165-70.

Gromer S, Eubel JK, Lee BL, et al. 2005. Human selenoproteins at a glance. Cell Mol Life Sci, 62:2414-37.

Guerra LN, Ríos de Molina Mdel C, Miler EA, et al. 2005. Antioxidants and methimazole in the treatment of Graves' disease: effect on urinary malondialdehyde levels. Clin Chim Acta, 352:115-20.

Hunter DJ, Morris JS, Stampfer MJ, et al. 1990. A prospective study of selenium status and breast cancer risk. JAMA, 264:1128-31.

Jakupoglu C, Przemeck GK, Schneider M, et al. 2005. Cytoplasmic thioredoxin reductase is essential for embryogenesis but dispensable for cardiac development. Mol Cell Biol, 25:1980-8.

Kim H, Park S, Suh JM, et al. 2001. Thyroid-stimulating hormone transcriptionally regulates the thiol-specific antioxidant gene. Cell Physiol Biochem, 11:247-52.

Kimura T, Okajima F, Sho K, et al. 1995. Thyrotropin-induced hydrogen peroxide producrion in FRTL-5 thyroid cells is mediated not by adenosine 3',5'-monophosphate, but by $\mathrm{Ca} 2+$ signalling followed by phospholipase-A2 activation and potentiated by an adenosine derivative. Endocrinology, 136:116-23.

Koenig RJ. 2005. Regulation of type 1 iodothyronine deiodinase in health and disease. Thyroid, 15:835-40.

Kohrle J, Jakob F, Contempre B, et al. 2005. Selenium, the thyroid, and the endocrine system. Endocr Rev, 26:944-84.

Kryukov GV, Castellano S, Novoselov SV, et al. 2003. Characterization of mammalian selenoproteomes. Science, 300:1439-43.

Leonard JL, Rosenberg IN. 2003. Thyroxine 5'-deiodinase activity of rat kidney. Observations on activation by thiols and inhibition by propylthiouracil. Endocrinology, 103:2137-44.

Levander OA, Beck MA. 1997. Interacting nutritional and infectious etiologies of Keshan disease: insights from Coxackie virus B-induced myocarditis in mice deficient in selenium or vitamin E. Biol Trace Elem Res, 56:5-21.

Matsui M, Oshima M, Oshima H, et al. 1996. Early embryonic lethality caused by targeted disruption of the mouse thioredoxin gene. Dev Biol, 178:179-85.

Mazopakis EE, Papadakis JA, Papadomanolaki MG, et al. 2007. Effects of 12 months treatment with L-selenomethionine on serum anti-TPO levels in patients with Hashimoto's thyroiditis. Thyroid, 17:609-12.

Moncayo R, Moncayo H. 2005. Nutritional treatment of incipient thyroid autoimmune disease. Influence of selenium supplementation on thyroid function and morphology in children and young adults. Clin Nutr, 24:530-1.

Negro R, Formoso G, Mangieri T, et al. 2006. Levothyroxine treatment in euthyroid pregnant women with autoimmune thyroid disease: effects on obstetrical complications. J Clin Endocrinol Metab, 91:2587-91.

Negro R, Greco G, Mangieri T, et al. 2007. The influence of selenium supplementation on postpartum thyroid status in pregnant women with thyroid peroxidase autoantibodies. J Clin Endocrinol Metab, 92:1263-8.

Nonn L, Williams RR, Erickson RP, et al. 2003. The absence of mitochondrial thioredoxin 2 causes massive apoptosis, exencephaly, and early embryonic lethality in homozygous mice. Mol Cell Biol, 23:916-22.

Poppe K, Glinoer D. 2003. Thyroid autoimmunity and hypothyroidism before and during pregnancy. Hum Reprod Update, 9:149-61.

Prummel MF, Wiersinga WM. 2004. Thyroid autoimmunity and miscarriage. Eur J Endocrinol, 150:751-5. 
Rayman MP. 2000. The importance of selenium to human health. Lancet, 356:233-41.

Seven R, Gelişgen R, Seven A, et al. 2001. Influence of propylthiouracil treatment on oxidative stress and nitric oxide in Basedow disease patients. J Toxicol Environ Health A, 62:495-503.

Smyth PP. 2003. Role of iodine in antioxidant defence in thyroid and breast disease. Biofactors, 19:121-130

St Germain DL, Hernandez A, Schneider MJ, et al. 2005. Insights into the role of deiodinases from studies of genetically modified animals. Thyroid, 15:905-16.

Stagnaro-Green A. 2004. Postpartum thyroiditis. Best Pract Res Clin Endocrinol Metab, 18:303-16.

Stawicki SP, Lyons M, Aloupis M, et al. 2007. Current evidence from phase III clinical trials of selenium supplementation in critically ill patients: why should we bother? Mini Rev Med Chem, 7:693-9.

Stranges S, Marshall JR, Natarajan R, et al. 2007. Effects of long-term selenium supplementation on the incidence of type 2 diabetes: a randomized trial. Ann Intern Med, 147:217-23.

Strieder TG, Prummel MF, Tijssen JG, et al. 2003. Risk factors for and prevalence of thyroid disorders in a cross-sectional study among healthy female relatives of patients with autoimmune thyroid disease. Clin Endocrinol, 59:396-401.

Sun QA, Kirnarsky L, Sherman S, et al. 2001. Selenoprotein oxidoreductase with specificity for thioredoxin and glutathione systems. Proc Natl Acad Sci USA, 98:3673-8.
Sun QA, Wu Y, Zappacosta F, Jeang KT, et al. 1999. Redox regulation of cell signalling by selenocysteine in mammalian thioredoxin reductases. J Biol Chem, 274:24522-30.

Surai P. 2006. Selenium in nutrition and health. Nottingham, UK: Nottingham University Press.

Vanderpas JB, Contempre B, Duale NL, et al. 1990. Iodine and selenium deficiency associated with cretinism in northern Zaire. Am J Clin Nutr, 52:1087-93.

Vinceti M, Wei ET, Malagoli C, et al. 2001. Adverse health effects of selenium in humans. Rev Environ Health, 16:233-51.

Wertenbruch T, Willenberg HS, Sagert C, et al. 2007. Serum selenium levels in patients with remission and relapse of Graves' disease. Med Chem, 3:281-4.

Whanger PD. 2002. Selenocompounds in plants and animals and their biological significance. J Am Coll Nutr, 21:223-32.

Xia Y, Hill KE, Byrne DW, et al. 2005. Effectiveness of selenium supplements in a low-selenium area of China. Am J Clin Nutr, 81:829-34.

Zhong L, Holmgren A. 2000. Essential role of selenium in the catalytic activities of mammalian thioredoxin reductase revealed by characterization of recombinant enzymes with selenocysteine mutations. J Biol Chem, 275:18121-8.

Zimmermann MB, Kohrle J. 2002. The impact of iron and selenium deficiencies on iodine and thyroid metabolism. Biochemistry and relevance to public health. Thyroid, 12:867-78. 
